\title{
Addressing metabolic heterogeneity in clear cell renal cell carcinoma with quantitative Dixon MRI
}

Yue Zhang, ${ }^{1}$ Durga Udayakumar, ${ }^{1,2}$ Ling Cai, ${ }^{3,4}$ Zeping Hu, ${ }^{3}$ Payal Kapur, ${ }^{5,6,7}$ Eun-Young Kho, ${ }^{3}$ Andrea Pavía-Jiménez, ${ }^{7,8}$ Michael Fulkerson, ${ }^{1}$ Alberto Diaz de Leon, ${ }^{1}$ Qing Yuan, ${ }^{1}$ Ivan E. Dimitrov, ${ }^{2,9}$ Takeshi Yokoo, ${ }^{1,2}$ Jin Ye, ${ }^{10}$ Matthew A. Mitsche, ${ }^{10}$ Hyeonwoo Kim, ${ }^{10}$ Jeffrey C. McDonald, ${ }^{10}$ Yin Xi, ${ }^{1}$ Ananth J. Madhuranthakam, ${ }^{1,2}$ Durgesh K. Dwivedi, ${ }^{1}$ Robert E. Lenkinski, ${ }^{1,2}$ Jeffrey A. Cadeddu, ${ }^{1,6}$ Vitaly Margulis, ${ }^{6}$ James Brugarolas, ${ }^{7,8}$ Ralph J. DeBerardinis, ${ }^{3}$ and Ivan Pedrosa ${ }^{1,2,7}$

${ }^{1}$ Radiology, ${ }^{2}$ Advanced Imaging Research Center, ${ }^{3}$ Children's Medical Center Research Institute, ${ }^{4}$ Quantitative Biomedical Research Center, ${ }^{5}$ Pathology, ${ }^{6}$ Urology, ${ }^{7}$ Kidney Cancer Program - Simmons Comprehensive Cancer Center, and ${ }^{8}$ Internal Medicine, University of Texas (UT) Southwestern Medical Center, Dallas, Texas, USA. 'P Philips Medical Systems, Cleveland, Ohio, USA. ${ }^{10}$ Molecular Genetics, UT Southwestern Medical Center, Dallas, Texas, USA.

BACKGROUND. Dysregulated lipid and glucose metabolism in clear cell renal cell carcinoma (ccRCC) has been implicated in disease progression, and whole tumor tissue-based assessment of these changes is challenged by the tumor heterogeneity. We studied a noninvasive quantitative MRI method that predicts metabolic alterations in the whole tumor.

METHODS. We applied Dixon-based MRI for in vivo quantification of lipid accumulation (fat fraction [FF]) in targeted regions of interest of 45 primary ccRCCs and correlated these MRI measures to mass spectrometry-based lipidomics and metabolomics of anatomically colocalized tissue samples isolated from the same tumor after surgery.

RESULTS. In vivo tumor FF showed statistically significant $(\boldsymbol{P}<0.0001)$ positive correlation with histologic fat content (Spearman correlation coefficient, $\rho=0.79$ ), spectrometric triglycerides ( $\rho$ $=0.56)$ and cholesterol $(\rho=0.47)$; it showed negative correlation with free fatty acids $(\rho=-0.44)$ and phospholipids $(\rho=-0.65)$. We observed both inter- and intratumoral heterogeneity in lipid accumulation within the same tumor grade, whereas most aggressive tumors (International Society of Urological Pathology [ISUP] grade 4) exhibited reduced lipid accumulation. Cellular metabolites in tumors were altered compared with adjacent renal parenchyma.

CONCLUSION. Our results support the use of noninvasive quantitative Dixon-based MRI as a biomarker of reprogrammed lipid metabolism in cCRCC, which may serve as a predictor of tumor aggressiveness before surgical intervention.

FUNDING. NIH R01CA154475 (YZ, MF, PK, IP), NIH P50CA196516 (IP, JB, RJD, JAC, PK), Welch Foundation I-1832 (JY), and NIH P01HL020948 (JGM).

Authorship note: $Y Z$ and DU contributed equally to the work

Conflict of interest: IED is an employee of Philips Healthcare and provided technical support

Submitted: March 29, 2017

Accepted: June 27, 2017

Published: August 3, 2017

\section{Reference information:}

JCI Insight. 2017;2(15):e94278.

https://doi.org/10.1172/jci.

insight. 94278

\section{Introduction}

Clear cell renal cell carcinoma (ccRCC) represents the most common subtype of RCC (65\%-70\%) and is highly variable in prognosis, biological behavior, and response to therapies (1-3). Sporadic ccRCC frequently carries an inactivating mutation of the von Hippel-Lindau $(V H L)$ tumor suppressor gene on chromosome $3 p$ with subsequent upregulation of the HIF/VEGF pathway, leading to intense tumor angiogenesis (4-6). The Tumor Cancer Genome Atlas (TCGA) analysis of gene and protein expression has confirmed major alterations in glucose, amino acid, and lipid metabolism in ccRCC, characterized histologically by prominent storage of glycogen and lipids $(7,8)$. High levels of lipid anabolic enzymes are expressed in ccRCC. In particular, fatty acid synthase (FASN; enzyme commission [EC] 2.3.1.85) and stearoyl-CoA desaturase 1 (SCD1; EC 1.14.19.1) are associated with poor prognosis $(9,10)$. Previous studies have also 


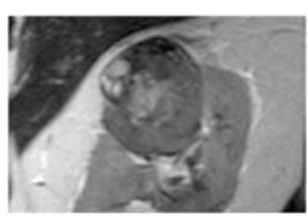

T2-Weighted

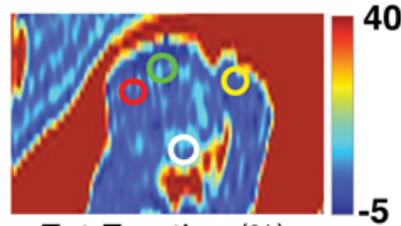

Fat Fraction (\%)

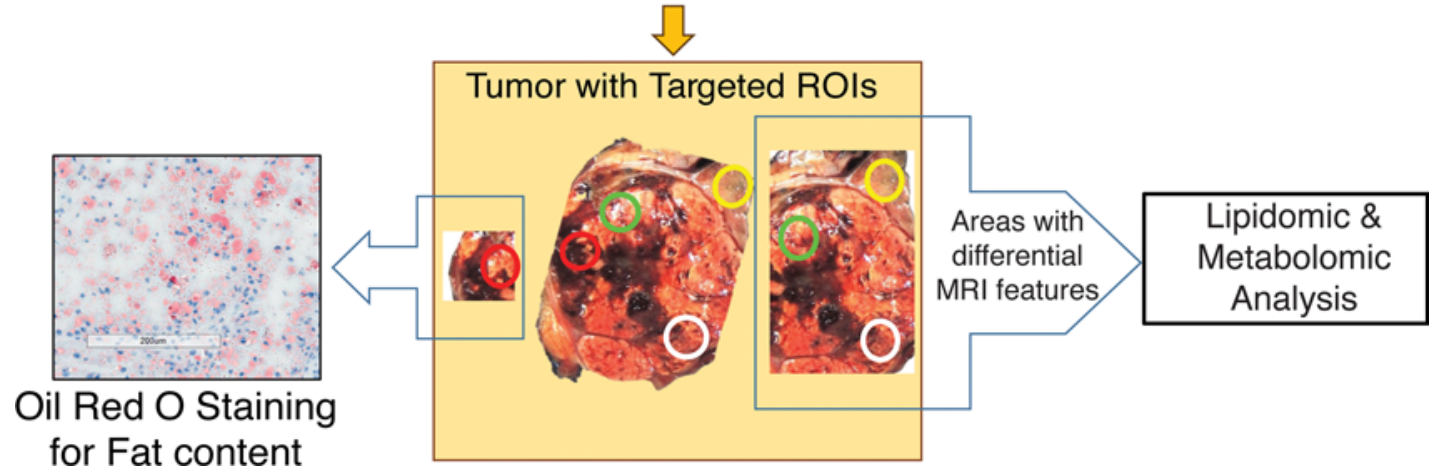

Figure 1. Anatomical coregistration of tissue-based analysis with MRI quantitative parameters. Patients underwent MRI, including a Dixon acquisition for fat fraction (FF) quantification. Colored circles representing the location and approximate size of the tissue samples were placed on representative locations within the tumor on the FF (\%) MRI maps. After surgical resection, each tumor was anatomically coregistered and sliced to match the imaging plane. Tissues from the same location in the tumor specimen as those of the targeted regions of interest (tROls) were collected as targeted samples. These samples underwent oil red $\mathrm{O}(\mathrm{ORO})$ staining for lipid distribution analysis and mass spectrometry for lipidomic analysis, as well as whole cell metabolite analysis. The results were then correlated with the two MRI measures. Tumors were classified based on the International Society of Urological Pathology (ISUP) grade.

shown increased accumulation of cholesterol in $\operatorname{ccRCC}(11,12)$. Altogether, these observations strongly suggest that reprogrammed lipid metabolism in ccRCC may provide not only biomarkers of oncological aggression, but perhaps therapeutic targets as well. While percutaneous tissue biopsies can offer a preoperative diagnosis of kidney cancer with high sensitivity, they are invasive and commonly yield inadequate results for tumor grade (13). Furthermore, the preoperative assessment of metabolic alterations in vivo is challenged by the intratumoral molecular heterogeneity that characterizes and likely drives the biological behavior of ccRCC (14). Moreover, the well-documented genetic branched evolution of ccRCC (15) would suggest that tumors may also undergo a temporal transformation of their metabolic phenotype as they evolve from more indolent, lower-grade neoplasms into aggressive, higher-grade tumors. The clinical implementation of active surveillance protocols and lack of established criteria for neoadjuvant therapy in ccRCC emphasize the need for diagnostic tools that can accurately capture the metabolic phenotype of a tumor in a given time. Thus, noninvasive quantitative imaging methods that provide an objective, spatially encompassing assessment of metabolic alterations in the whole tumor in vivo are appealing.

Multi-echo Dixon-based MRI (mDixon) (16) is a method for in vivo fat quantification that has been validated in liver steatosis against tissue biopsies (17-19). The purpose of this study was to prospectively assess the role of Dixon-based MRI-derived quantitative measures of intratumoral lipid accumulation as a noninvasive in vivo biomarker of heterogeneous metabolic reprogramming in ccRCC.

\section{Results}

Anatomical coregistration of tissue-based analysis with MRI quantitative parameters. We applied Dixon-based MRI for tumor lipid accumulation in targeted regions of interest (tROIs) in patients who underwent preoperative MRI imaging. Figure 1 represents the schematic of the experimental design and the workflow of this study. Patients underwent MRI, and quantitative estimates of fat fraction (FF) were derived for lipid phenotype as indicated. Patients then underwent radical or partial nephrectomy. The surgical specimen was spatially colocalized with the MRI and bivalved immediately after surgery (see Methods). Tissue samples were collected from anatomic locations within the tumor corresponding to the tROIs and subjected to histopathological staining, mass spectrometric analysis for lipid contents, and whole cell metabolite analysis. The readouts of tissue-based analysis were correlated with the quantitative MRI measures of lipid accumulation (FF). Each tumor was classified based on the International Society of Urological Pathology (ISUP) 
A

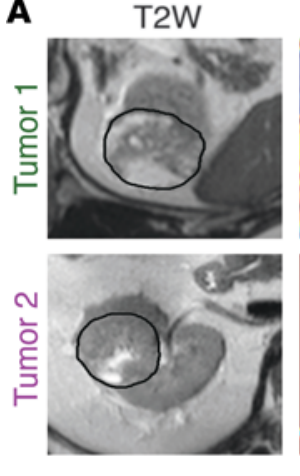

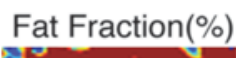
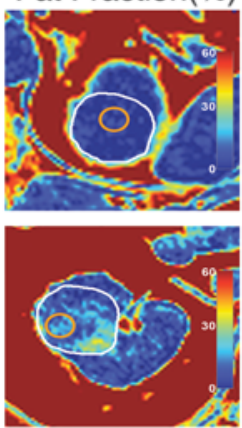

C
Oil Red O
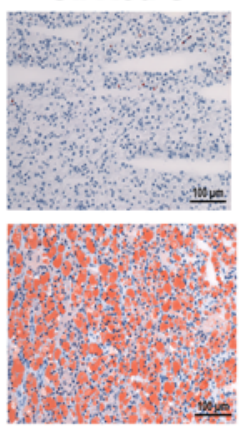

${ }^{30}\left[\begin{array}{cc}\hline \text { ISUP Grade } \\ \bullet & 2 \\ \square & 3 \\ 4 & 4 \\ \hline\end{array}\right.$
B $\begin{array}{r}20 \\ 18 \\ \bigcirc \\ \circ \\ \circ\end{array}$

$\circ$

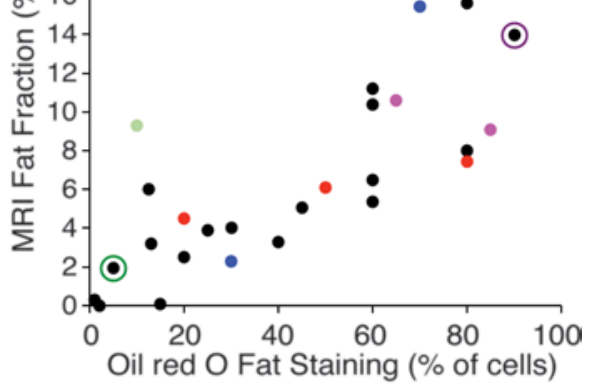

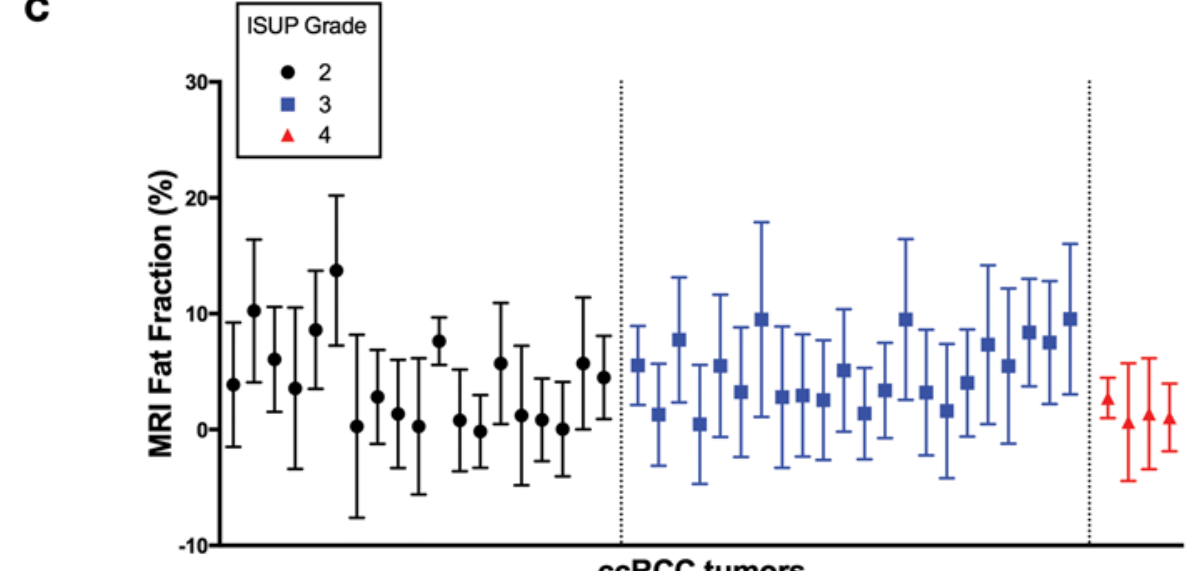

ccRCC tumors

Figure 2. Clear cell renal cell carcinoma (ccRCC) tumors exhibit heterogeneous lipid accumulation, independent of tumor grade. (A) Representative T2-weighted MRI images (left) and quantitative fat fraction (FF) maps (middle) of 2 tumors (black and white circular contours on both sets of images, respectively) indicating location of targeted region of interest (tROI, orange circle), and corresponding ex vivo oil red $\mathrm{O}$ (ORO) staining for fat content of targeted tumor samples as described in Methods (top panel: Tumor 1, ISUP grade 2; bottom panel: Tumor 2, ISUP grade 3). Color bars in FF maps indicate the percent of fat signal in tumor from $0 \%-60 \%$. The scale bar values in the $0 R 0$ staining indicate $100 \mu \mathrm{m}$. (B) Spearman correlation analysis between in vivo mean tumor $\mathrm{FF}$ and percentage of cells staining with $\mathrm{ORO}$ in 27 targeted tumor samples $(\rho=0.79, P<0.0001)$. Green circle, Tumor 1 in $\mathbf{A}$ ( $F F=2 \%$, ORO percentage $=5 \%)$; purple circle, Tumor 2 in $\mathbf{A}(F F=14 \%$, ORO percentage $=90 \%)$. Same-colored data points represent tissue samples obtained at different locations within the same tumor. (C) Distribution of FF across tumor grades from 45 ccRCCs. The $x$-axis is sectioned in 3 parts representing International Society of Urological Pathology (ISUP) grade, with each point representing 1 individual tumor. The $y$-axis represents the mean percentage of FF in the whole tumor as measured by Dixon MRI. Vertical whiskers represent the mean \pm SD within the individual tumor, indicating substantial intratumoral FF heterogeneity. ccRCCs exhibit heterogeneous intratumoral lipid accumulation among different tumor grades and within each tumor grade. Grade-4 tumors exhibited lower fat content than grade-3 tumors $(P=0.0163)$, although not different than grade- 2 tumors $(P=0.33)$.

nucleolar grade (20). The patient and tumor characteristics for the ccRCC tumors collected and analyzed in this study are provided in Supplemental Table 1 (supplemental material available online with this article; https://doi.org/10.1172/jci.insight.94278DS1).

MRI FF quantification correlated with intracellular lipids and indicated heterogeneity in lipid accumulation in ccRCCs. Since ccRCC tumors show histopathologically abundant storage of lipid species (21-23), a validated noninvasive methodology to assess the heterogeneity of lipid accumulation in these tumors would be a valuable tool. Hence, we used an established methodology, mDixon, originally developed for quantitative evaluation of liver steatosis (24). We generated an FF tumor map to correlate with histopathological and molecular lipid quantity. First, we validated MRI-derived FF as a surrogate of intratumoral fat content by comparing it with the results of oil red O (ORO) stain, a diazo dye used for staining triglycerides (TG), and cholesterol esters (CE) $(25,26)$. Freshly frozen tissues were stained and manually scored by an urologic pathologist as percentage of positively stained tumor cells. Figure 2A shows representative images of tumors with tROIs exhibiting low fat content (tumor 1) and high fat content (tumor 2), along with the colocalized ORO stains of the targeted tissues samples obtained from the same locations in both tumors. Figure 2B represents the scatter plot derived from 27 targeted tumor samples (22 patients) stained for ORO and the corresponding $\mathrm{FF}$ values derived from $\mathrm{tROIs}$. The $\mathrm{FF}$ values from $\mathrm{tROIs}$ showed significant positive correlation 
A

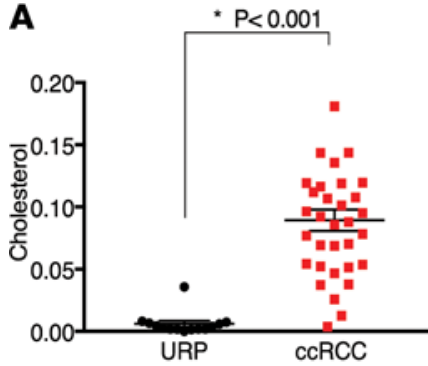

$* P<0.001$

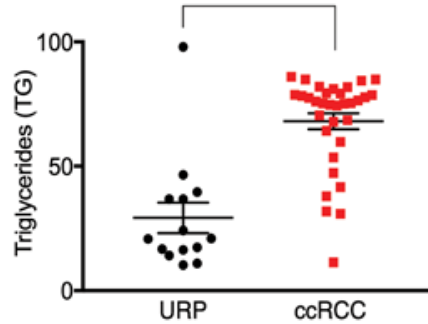

${ }^{*} \mathrm{P}<0.001$

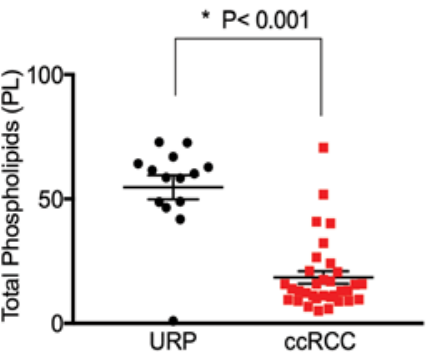

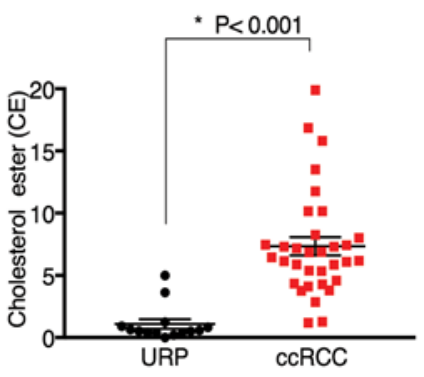
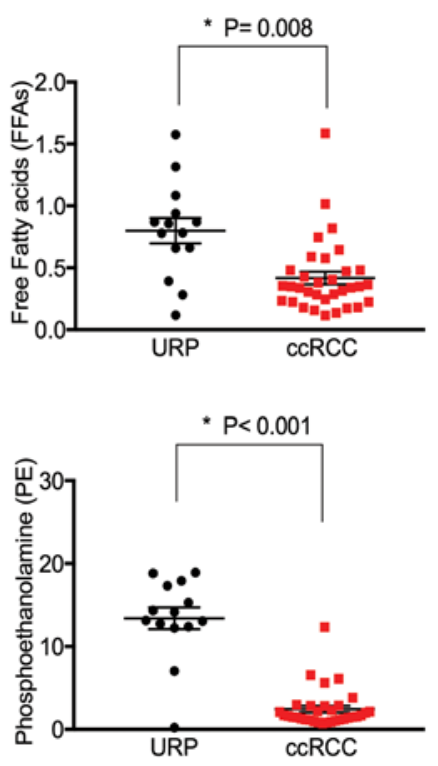

B
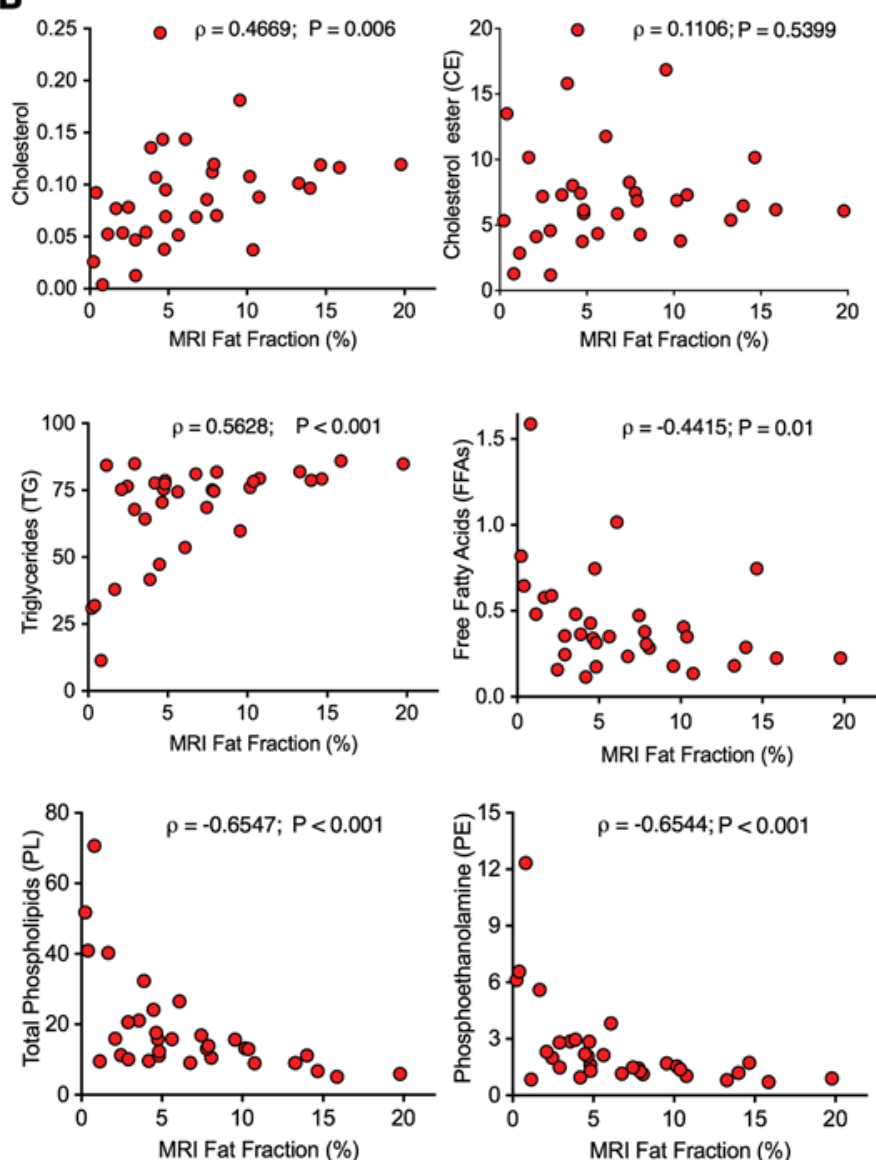

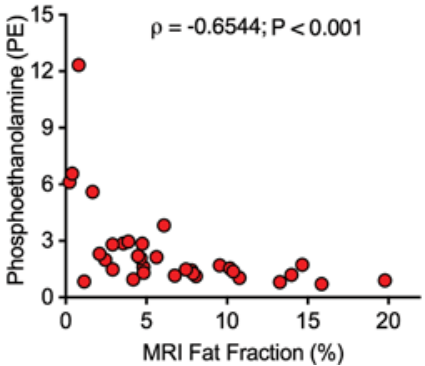

Figure 3. Lipidomic profiling of clear cell renal cell carcinoma (ccRCC) tumors showed differential levels in the uninvolved renal parenchyma (URP) and ccRCC tissue samples and correlated with fat fraction measures. (A) The data represent the normalized intensity peaks of selected lipid species, with the horizontal bars and whiskers indicating the means \pm SEM in cCRCC and URP samples ( $n=14$ patients). The intensity of each peak was normalized to the total lipid signal and summed to give the intensity of each class as percentage of all identified lipids. The statistical significance of the differences between measurements as indicated in the figure were assessed using an unpaired Mann-Whitney $U$ test. (B) Correlation of fat fraction (FF) assessment using Dixon-based MRI method with lipidomic analysis of 33 targeted tumor samples ( $n=14$ patients) from the same tumor location. Levels of lipid species were analyzed in fresh targeted tumor samples by mass spectrometry, as indicated in Methods. The Spearman correlation was applied to evaluate correlation between fat fraction and levels of cholesterol $(\rho=0.47, P=0.006)$, cholesterol ester $(\rho=0.11, P=0.5399)$, triglycerides $(\rho=0.56, P<0.001)$, free fatty acids $(\rho=-0.44, P=0.01)$, total phospholipids $(\rho=-0.65, P<0.001)$, and phosphatidylethanolamine $(\rho=-0.65, P<0.001)$.

with the percentage of tumor cells staining positively for ORO (Spearman correlation coefficient, $\rho=0.79$; $P<0.0001$; after adjusting for multiple measures in the same tumor, $\rho=0.79 ; 95 \%$ bootstrap CI, $0.69-0.87$ ). The linear regression model showed $\mathrm{FF}=0.13 \times \mathrm{ORO} \%(95 \% \mathrm{CI}$ of the slope is $0.09-0.18, P<0.0001$; after adjusting for multiple measurements in the same tumor, $0.13 ; 95 \%$ CI, $0.08-0.19, P=0.0029$ ). The tumor characteristics of this subset of tumors are provided in Supplemental Table 2. Importantly, Dixon-MRI was able to capture intratumoral heterogeneity in lipid accumulation with different samples from the same tumor exhibiting distinct FF levels (Figure. 2B). Since Dixon-MRI is designed to measure tissue TG, our results are supported by previous reports indicating that ORO stains predominantly TG and cholesteryl oleate (27). This further validated the FF quantification methodology to assess the lipid distribution in our ccRCC samples. Since the ccRCC phenotype is characterized by dysregulated lipid biosynthesis and this has been associated with tumor prognosis (7) and invasiveness (23), we evaluated the relationship between MRI-derived $\mathrm{FF}$ and tumor grades in $45 \mathrm{ccRCC}$, as defined by ISUP grading. We found heterogeneous lipid accumulation not only between the tumors of different grade, but also between tumors within the same tumor grade (Figure $2 \mathrm{C}$ ). The intratumoral heterogeneity is illustrated by the $\mathrm{SD}$ of the FF measures for any given tumor, with several tumors exhibiting a range of $\mathrm{FF}$ varying over $10 \%$ in different areas of the tumor; the range of mean $\mathrm{FF}$ across all tumors was $-0.2 \%$ to $14 \%$. However, analysis of multiple systematic ROIs (sROIs) obtained on each individual tumor (see Methods) demonstrated that $63 \%$ of the variation in FF was due to 
A

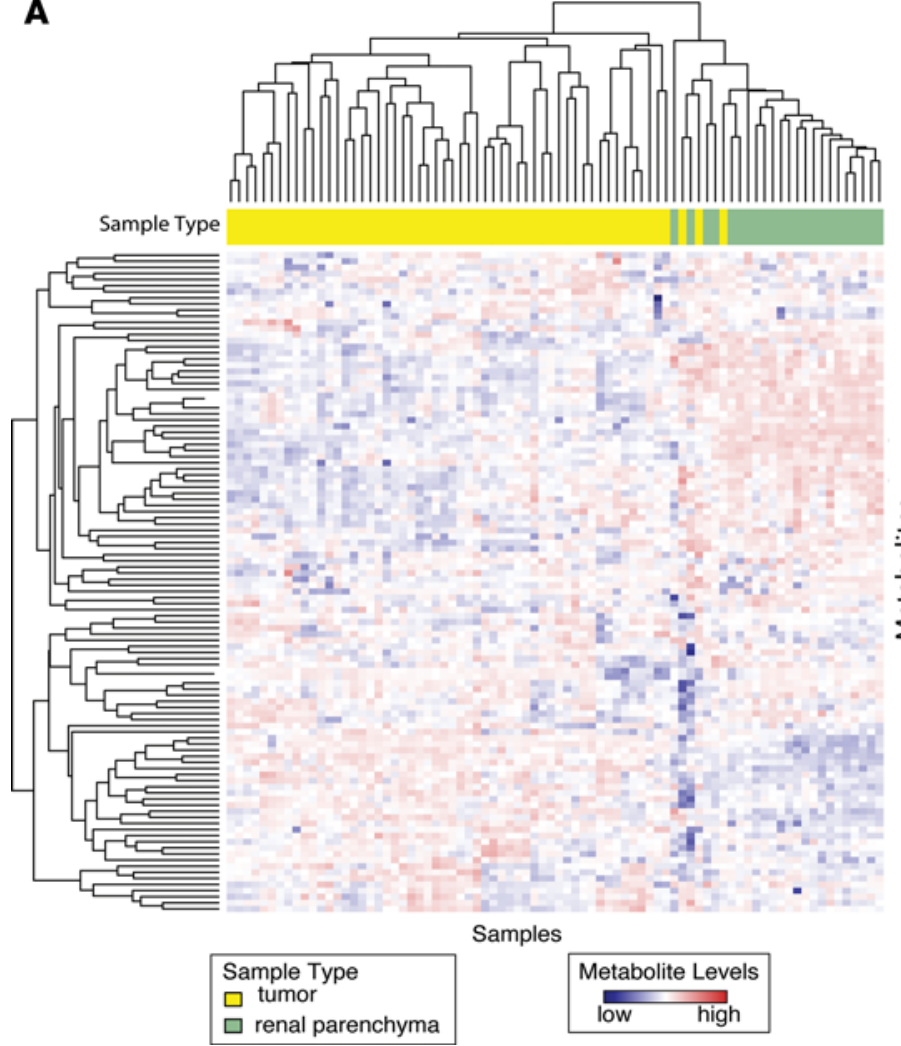

B

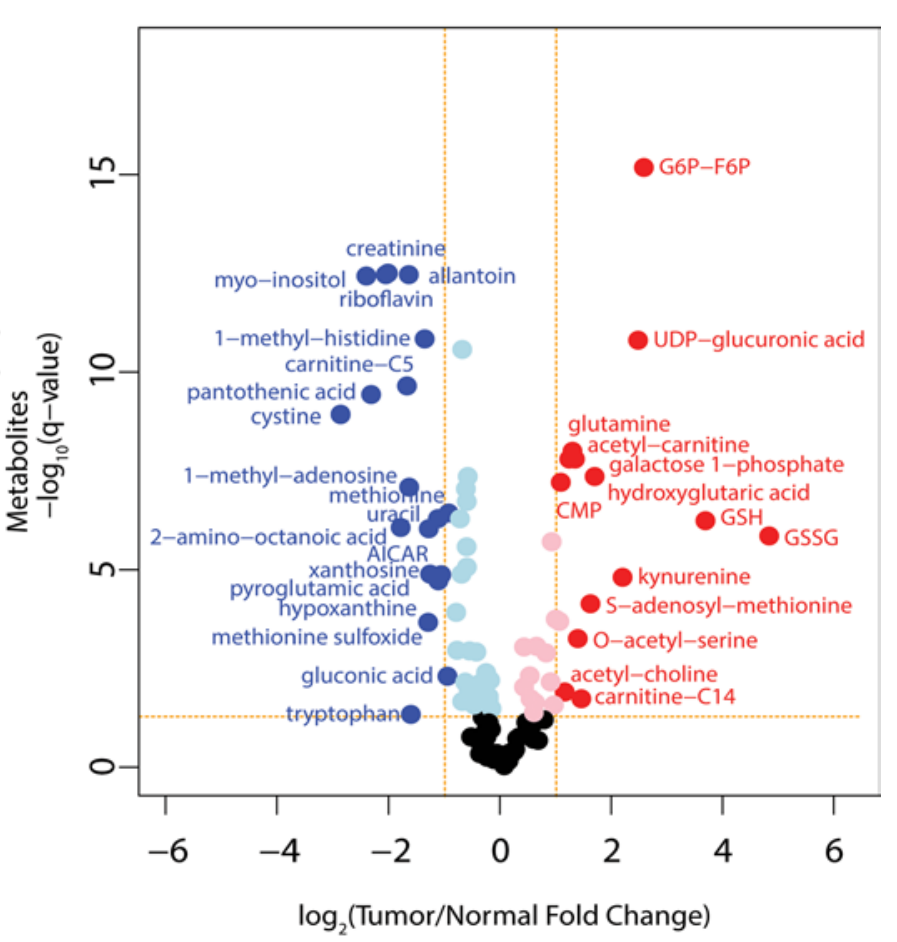

Figure 4. Clear cell renal cell carcinoma (ccRCC) exhibits widespread metabolic alterations compared with uninvolved renal parenchyma (URP). (A) Heatmap of unsupervised hierarchical clustering of 80 human samples in 23 patients with cCRCC ( 57 tumors and 23 URP) analyzed for the abundance of 108 metabolites. Each column and row corresponds to 1 tissue sample and a single metabolite, respectively. Yellow cluster represents tumor samples, and the green cluster represents normal samples. (B) Volcano plot analysis of metabolites significantly altered in tumor versus URP. The fold change of metabolite levels is plotted on the $x$-axis. The FDR-adjusted significance is plotted on the $y$-axis. Horizontal dash line represent $q$ value cutoff of -log ${ }_{10}$ (0.05); vertical dash lines represent $\log _{2}$-fold change (tumor/URP) of -1 and +1.71 . Metabolites with significantly different levels in tumor vs. URP samples are indicated as blue, light blue, pink, and red dots. With fold-change cutoff of 2, 19 metabolites were decreased (blue) and 14 metabolites were increased (red) in tumor vs. URP samples. Black dots represent the metabolites that did not pass the $q$ value cut-off. A two-sided $t$ test was performed, and the hits with $P<0.05$ adjusted by Benjamini-Hochberg procedures were considered significant.

intertumor variation (intraclass correlation $[\mathrm{ICC}]=0.63$ ), whereas $37 \%$ of variability represents intratumoral heterogeneity in lipid accumulation. Furthermore, there was no difference in intratumoral heterogeneity in FF based on tumor size for all tumors $(P>0.05)$ or between those within the same ISUP grade (ISUP $2,3$, or $4 ; P>0.05)$. Overall, these data indicate that the heterogeneity in lipid content is greater between different tumors than the intratumoral heterogeneity. Lipid accumulation was statistically lower in grade-4 tumors than in grade- 3 tumors $(P=0.0299)$, although not in grade- 2 tumors $(P=0.0787)$. Our results were consistent with the previous observation that high-grade ccRCC has lower lipid content (28).

MRI FF quantification predicts altered fatty acid metabolism. Next, we evaluated whether our ccRCC tissue samples exhibited altered fatty acid metabolism, one of the characteristic biochemical signatures of cancer cells $(21,23)$. Global lipidomic profiling using our nontargeted assay platform helped detect 1,759 lipid species in the ccRCC samples (Supplemental Table 3). These lipid molecules included phospholipids (PL; 980 species), sphingolipids (74 species), neutral lipids (677 species), and polar lipids (28 species). We observed a statistically significant $(P<0.0001)$ increase in the TG and CE levels in our ccRCC samples as compared with the uninvolved renal parenchyma (URP), a finding consistent with the literature (29-31) (Figure 3A). In addition to the accumulation of CE and TG, our experiments also showed ccRCC-associated alterations in phosphoethanolamine (PE) (Figure 3A). We observed a lower level of PE in the tumor samples compared with samples of URP, consistent with a previous report (29), further suggesting that the $\mathrm{PE}$ pathway downregulation is another significant feature of ccRCC tumors. We then determined whether the MRI-derived FF values correlated with altered lipid metabolic features we observe in these ccRCC tumors. The FF values derived from the tROIs corresponding to tissues used for lipid analysis (Figure 3B) 
A

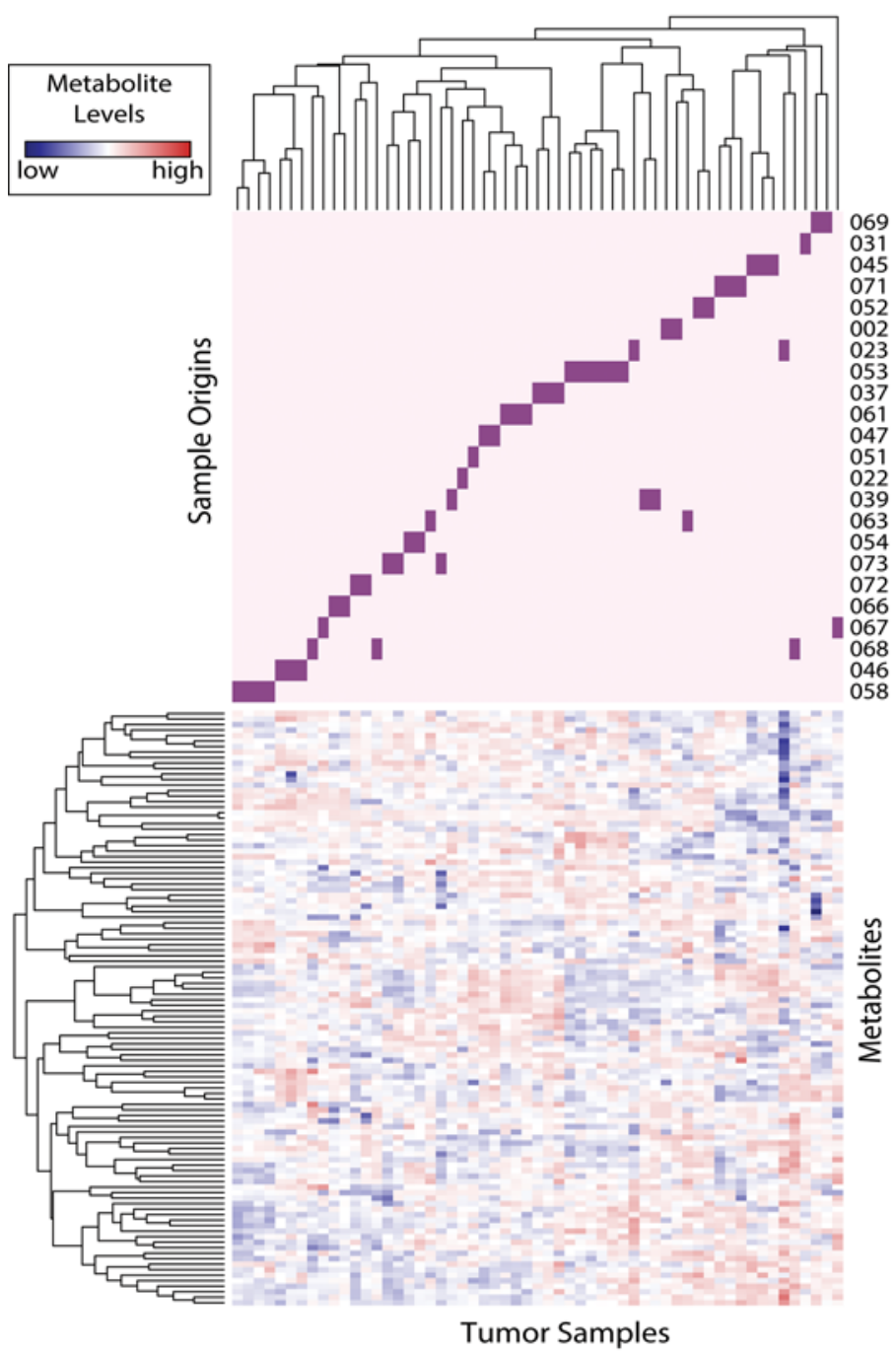

B

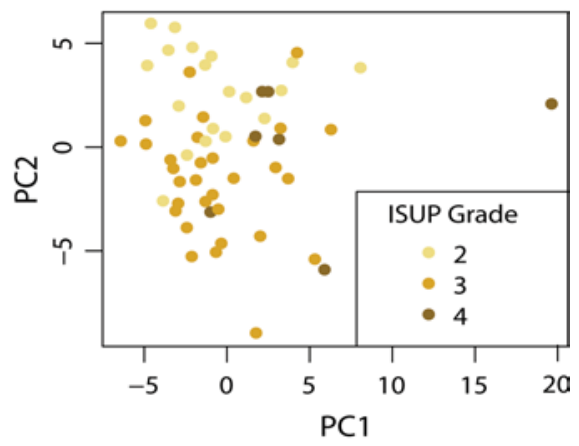

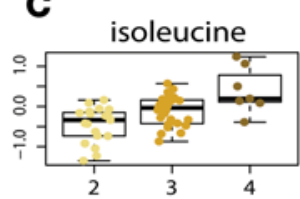

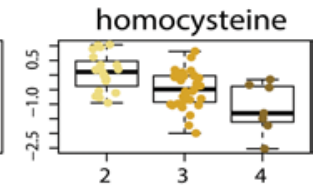

proline

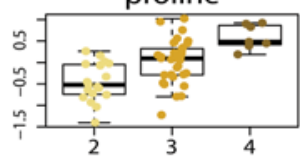

glycine
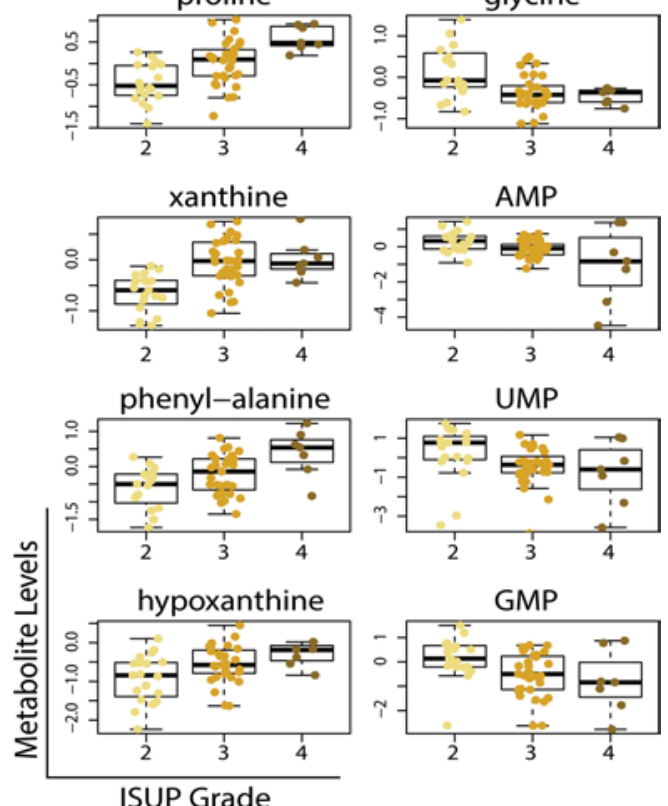

ISUP Grade

Figure 5. Clear cell renal cell carcinoma (ccRCC) exhibit greater intertumoral than intratumoral metabolic heterogeneity. (A) Heatmap for the metabolomics analysis of 57 ccRCC samples (from 23 patients) ordered by average linkage hierarchical clustering. Columns represent tumor samples, and rows represent metabolites. Colored rows above the heatmap indicate the origin of the samples, with the tumor identification number in the $y$-axis. Tumor samples obtained from the same tumor tend to cluster, except for a few tumors. (B) Metabolomics data from principal component analysis (PCA) of samples with different International Society of Urological Pathology (ISUP) grades indicates lack of differentiation based on the overall tumor metabolic profile. (C) Graphical representation of ISUP grade-dependent variations of the top metabolites (adjusted $P<0.05$ ) showing increase $(n=5)$ or decrease $(n=5)$ in expression. A two-sided Jonckheere-Terpstra test (Jonckheere trend test) was performed. $P<0.05$ adjusted by Benjamini-Hochberg procedures. The error bars represent the mean \pm SD of the indicated metabolite levels of the tumors within each grade.

showed a positive Spearman correlation with increased TG $(\rho=0.56, P<0.001)$ and free cholesterol $(\rho=$ $0.47, P=0.006)$, and a weak negative correlation with the free fatty acids (FFA) $(\rho=-0.44, P=0.01)$. Interestingly, we observed an indirect correlation with PL. Specifically, we found a negative correlation between FF in the tumors and PE, a predominantly membrane-localized lipid (Figure 3B, see Discussion). Hence, Dixon-MRI can be applied to noninvasively predict the abundance and alterations directly in intracellular lipids and indirectly in membrane-bound lipids, which are both linked to ccRCC tumor progression. It has been reported that cancer cells selectively activate cholesterol synthesis pathways to keep up with energy demand and for survival $(32,33)$, and increased activity of lipogenic enzymes such as FASN is a known phenotype in ccRCCs $(7,9)$. Additionally, FASN expression predicts poor prognosis in cancer patients 


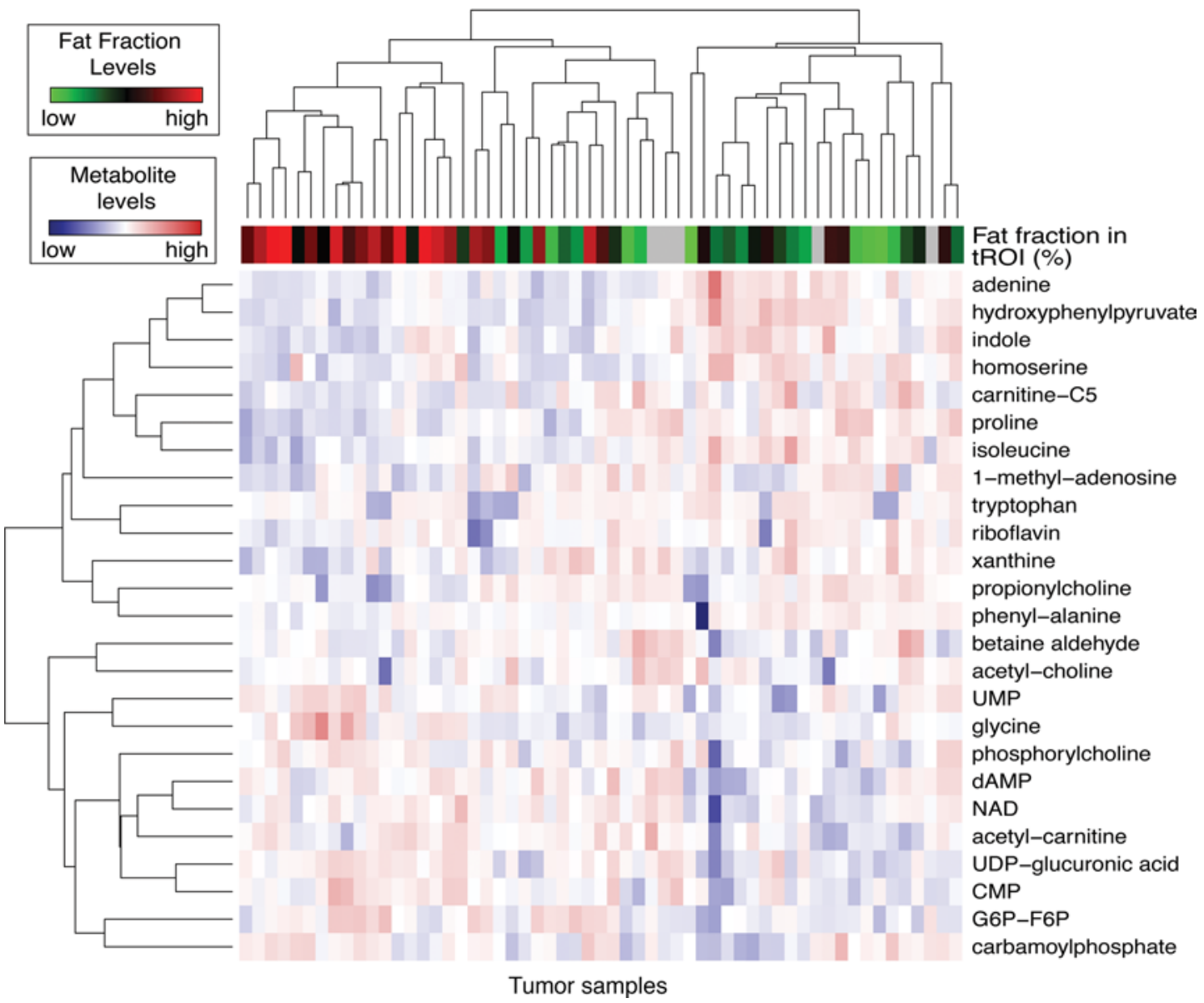

Figure 6. Dixon MRI measured fat fraction correlates with altered metabolomic features in clear cell renal cell carcinoma (ccRCC). Heatmap shows selected metabolites that positively or negatively correlate with fat fraction levels in 57 targeted ccRCC samples in 23 tumors. The quantitative measure of fat fraction as a percentage of the total MRI signal within a targeted region of interest (tROI) corresponding to the anatomically coregistered tumor sample for each ccRCC tumor is indicated as a color scale above the heatmap. A cutoff of nominal $P<0.05$ was used to select metabolites from Spearman's rank correlation between metabolites and fat fraction values.

in general (34), and specifically in ccRCC patients (7). We investigated whether the FF correlated with the FASN expression in tumors. Immunohistopathological staining was done on $21 \mathrm{ccRCC}$ tumors, and cytoplasmic FASN expression was analyzed and scored using the automated Aperio Imagescope software. Although, we did not observe a significant correlation, likely due to known outliers (see Discussion), FASN expression showed a positive trend toward correlation with FF values (Supplemental Figure 1).

ccRCC tumor-specific metabolic alterations and intertumoral heterogeneity. ccRCC is characterized by significant alterations in intermediary metabolism and antioxidant response, and metabolite increases in glutathione and cysteine/methionine metabolic pathways are associated with tumor progression and metastasis $(35,36)$. In order to understand the correlation between $\mathrm{FF}$ and cellular metabolism in these tumors, we performed a targeted metabolomic analysis of ccRCC, from fresh frozen high-quality tumor samples and corresponding adjacent URP. This cohort consisted of 80 samples (57 ccRCC tumors and 23 normal tissues) across different ISUP grades. Mass spectrometric analysis (from glycolysis, the pentose phosphate pathway, one-carbon/nucleotide metabolism, the TCA cycle, amino acid degradation, and other pathways) detected 108 metabolites, where each metabolite was normalized to the total protein content and relative abundance was determined between the samples. Unsupervised clustering of metabolite levels largely discriminated between URP and tumors (Figure 4A). Volcano plot analysis of metabolites significantly altered in tumor versus URP is shown in Figure 4B. The fold change of metabolite levels is plotted on the $x$-axis, and the FDR-adjusted significance is plotted on the $y$-axis. Many metabolites decreased in ccRCC by half or more as compared with URP samples with stringent and nominal cutoffs, as indicated in the Figure 4 legend. Further, we identified significant increase in some metabolites in $\operatorname{ccRCC}(q<0.05)$ 
by 2 -folds or more than URP samples (Figure 4B). Interestingly, we found a significant increase in the carbohydrate pathway metabolites (glucose 6-phosphate [G6P], and galactose 1-phosphate (Gal1P) and redox markers (glutathione [GSH] and glutathione disulfide [GSSG]), which are consistent with prior metabolic analysis reported on ccRCC tumors (35-37).

Our data results confirm stricking metabolic differences between ccRCC and adjacent renal parenchyma, a finding that is consistent with previously reported metabolic alterations in these tumors, and support using these tissue-based analyses for correlation with in vivo MRI measures of FF.

To further understand the metabolic heterogeneity between different ccRCC tumors (intertumor), and also between different tissue samples from the same tumor (intratumor), an analysis ordered by average linkage hierarchical clustering was performed. Figure 5A shows the heatmap for the metabolomic analysis of 57 ccRCC samples (from 23 patients), where columns represent tumor samples and rows represent metabolites. Individual fragments were metabolomically unique, but fragments from the same tumor generally clustered together, indicating that intratumor heterogeneity is exceeded by heterogeneity among tumors from different patients (Figure 5A and Supplemental Table 4). Although principal component analysis (PCA) of samples from different tumor grades (Figure 5B) did not indicate a clearly defined differentiation based on the overall tumor metabolic profile, there were a few metabolites that exhibited significant increased or decreased levels ( $n=5$ in each group, adjusted $P<0.05$ ) among different tumor grades (Figure $5 \mathrm{C}$ ).

MRI-based phenotyping detects intratumoral heterogeneity in altered metabolic states in ccRCC. Some metabolic features in human tumors can be predicted by MRI (38). We therefore tested whether our Dixon-based MRI FF values correlated with the metabolic features observed above. Figure 6 shows the heatmap of correlation profile between the FF values derived and the metabolites corresponding to those samples in 57 targeted ccRCC samples (from 23 patients). We obtained a panel of 25 metabolites with a cut-off of nominal $P<0.05$ (Supplemental Table 5). A number of amino acids (proline, isoleucine, and tryptophan) negatively correlated with the FF, suggesting a link between abundance of TG and CE in these tumors and alteration in amino acid metabolism. Other metabolites such as UMP, glycine, G6P-fructose 6-phosphate (G6P-F6P), and carbomoylphosphate exhibited a strong positive correlation with the FF values. However, several of these metabolites correlated with tumor ISUP grade (Figure 5), as well, possibly indicating a metabolic pathway independent of lipid metabolism.

\section{Discussion}

The invasive nature and limitations associated to percutaneous biopsies (e.g., small sample size) in ccRCC, an inherently heterogeneous disease, strengthens the value of accurate whole-tumor evaluation by imaging methodologies. Current methods for presurgical risk stratification of localized primary RCCs have limitations with up to $30 \%$ of these patients developing a recurrence in spite of optimal surgical resection (i.e., negative margins) (39). Hence, a reliable noninvasive imaging method to predict molecular, genetic, and metabolic alterations in the whole tumor may facilitate the management of ccRCC patients, the prediction of tumor aggressiveness, and the delivery of personalized care by optimizing therapeutic protocols based on the anticipated biologic behavior of any given tumor $(40,41)$. In this context, MRI is particularly well suited to provide a comprehensive phenotype characterization of ccRCCs based on excellent soft-tissue contrast and spatial resolution, as well as functional and quantitative techniques (41). In this study, we utilized Dixon-based MRI FF quantification as a platform to assess noninvasively signature metabolic features of ccRCC.

The Dixon-based MRI method utilizes a chemical shift technique to detect mobile lipid molecules (24), and our results confirm that is able to measure one of the histological metabolic hallmarks of the ccRCC subtype: the presence of intracytoplasmic vacuoles containing lipids called lipid droplets. These lipid droplets consist of a neutral lipid core containing TG and CE surrounded by a PL monolayer and associated lipid droplets surface proteins (42). The presence of lipid droplets results in decreased signal intensity on T1-weighted (T1W), opposed-phase images compared with the in-phase images of actively proliferating tumor areas. Previous reports have indicated a moderate-to-high sensitivity (42\%-82\%) and very high specificity (94\%-100\%) for the diagnosis of ccRCC among different renal masses when using a simplified 2-point Dixon approach for detecting intratumoral lipids $(43,44)$. Furthermore, the development of state-of-the-art multi-echo, multi-peak Dixon techniques such as the one used in our study allows for accurate quantification of fat tissue content (45). However, validation of Dixon-based MRI FF measurements in tumors against histopathology is lacking. To our knowledge, our study compared, for the first time, the MRI-based FF measures with histological lipid quantification in the same 
tumor region in a 45-ccRCC patient cohort. The simultaneous validation of the FF measurements against ORO histological lipid staining demonstrated strong significant correlation between FF and the endogenous lipid accumulation in tumor samples. Furthermore, we used the lipidomic approach, an emerging method used to obtain general lipid profiles, to determine whether the FF measures correlate with lipidomic alterations in our ccRCC patient cohort. In agreement with reported findings in the literature (29), we clearly showed signature lipid alterations in our tumor cohort (higher TG and CE, and lower levels of PL and PE) compared with URP. The significant correlation of FF against TG and $\mathrm{CE}$ indicate that the FF measures can predict alterations in lipid metabolites associated with tumor progression in vivo. However, Figure 3B indicates that some tumor samples with low FF levels in vivo had elevated TG on tissue-based analysis. To what extent this is the result of the precision of Dixon-based methods employed in our analysis, estimated to be $\pm 3 \%$ (46) versus intratumoral heterogeneity and/ or poor colocalization of the image-based analysis with the tissue sample, is unknown. Moreover, the shot-gun lipidomic analysis used in our study was chosen to interrogate possible correlations over a large number of lipids; however, direct targeted measurement of TGs in tumors may be needed for more accurate correlations with in vivo FF (47). These inconsistencies deserve further investigation.

We also observed a significant negative correlation between $\mathrm{FF}$ and $\mathrm{PE}$, another recently reported key lipidomic feature of ccRCC associated with tumor growth and proliferation (32). It is important to emphasize that MRI signal is derived from the mobile intracellular lipids (predominantly TG), not from the lipids in the membranes, which are essentially invisible to the Dixon MRI technique. Our results are in concordance with reported alterations in genetic pathways associated with increases in TG. Leonardi et al. (48) demonstrated that disruption of the synthesis pathway of $\mathrm{PE}$ in mice liver by inactivation of the gene PE cytidylyltransferase (ECT), which catalyzes the rate-limiting step of PE synthesis, leads to massive accumulation of TG in the lipid droplets in hepatocytes. The demonstration of a correlation between fat accumulation in vivo with MRI and the increased TG/decreased PE on lipidomic analysis in the same area of the tumor would support the knowledge that fat accumulation in ccRCC is the result of dysregulation in the PE metabolic pathway.

Our results indicate a statistically significant decrease in FF in ISUP grade- 4 tumors as compared with grade-3 tumors, although not with grade-2 tumors (Figure $2 \mathrm{C}$ ). This is consistent with previous reports indicating lower fat content in higher-grade tumors (28). However, the FF in low-grade tumors (ISUP grade 2) was not statistically significantly different than the combined FF of high-grade tumors (ISUP grades 3 and 4), largely due to substantial variation in FF values within grade-2 and -3 tumors, with some exhibiting abundant lipid content while others had no lipid accumulation at all. Although there is extensive evidence of the association between tumor grade and prognosis, the estimated disease-free survival after surgical resection of grade- 2 renal cancer varies between $66 \%-90 \%$ for pT2 and $56 \%-85 \%$ for pT3-stage tumors (49). These data indicate that the risk of metastatic disease in a priori more indolent tumors is still substantial. The marked variability of lipid accumulation in grade- 2 tumors in our study is intriguing. Based on these findings, we postulate that the role of Dixon-based MRI in the prediction of oncologic outcomes in ccRCC, including low-grade tumors, deserves attention. The ability to noninvasively detect a metabolic signature associated to grade- 4 tumors could be also of value in active surveillance protocols where loss of intratumoral accumulation of fat (i.e., initially detected on a lower-grade tumor) would be indicative of a possible transformation of the tumor toward a more aggressive phenotype. This could be particularly useful in the implementation of active surveillance protocols for larger, heterogenous tumors (e.g., cT1b disease, $>4 \mathrm{~cm}$ in size), which has been proposed as a reasonable option in select patients with significant comorbidities (50). Moreover, given the intratumoral heterogeneity of FF measures, a better tumor characterization may be achieved by directing percutaneous biopsies toward specific areas of the tumor with lower FF or those where a decrease in FF is documented over time.

Our results are also consistent with previous reports indicating that ccRCCs are metabolically heterogeneous. Indeed, we found that heterogeneity was more prominent among different tumors than within individual tumors, although our results clearly indicate some intratumor heterogeneity, as well. These findings were similar to the reported heterogeneity in non-small cell lung cancer patients receiving ${ }^{13} \mathrm{C}$-glucose infusions (38). Furthermore, analysis of MRI-measured lipid content also indicated greater heterogeneity among different tumors than within each tumor. Comparison of in vivo FF levels against cellular metabolites provided us with a panel of altered metabolites (Figure 6). However, these metabolites did not seem to cluster into specific metabolic pathways. Therefore, our data does not clarify the mechanism for fat accumulation in ccRCC. 
Our study has some limitations. First, based on tissue availability, ORO staining was not performed in all tumor samples. Furthermore, the FF were calculated with methodology developed and validated for liver steatosis where the $\mathrm{R} 2$ * of the various lipids were well understood. It is possible that the lipid peaks in ccRCC may have different R2*. However, a single R2* fitting for all hepatic lipids provides robust measurements of FF (51), and we found a strong correlation between FF and histologic accumulation of fat (i.e., ORO\%) within the available samples. Second, although every attempt was made to coregister the FF area with tissue specimens with the use of fiducial markers, this process can lead to sampling errors that can affect the correlations between FF and ORO percentage and lipidomic profile. Future studies will be focused on addressing these challenges by utilizing 3D-printed molds of the tumor for coregistration of the tissue specimen with the MR images (52), which we anticipate will increase the accuracy of sampling in the targeted ROIs within each patient. Third, although we found a statistically significant reduction in FF in ISUP grade- 4 tumors compared with ISUP grade- 3 tumors, the number of ISUP grade- 4 ccRCCs in our cohort was small; therefore, this preliminary finding needs to be evaluated in larger studies.

In conclusion, this set of rigorous validation of the Dixon-based MRI method against histology, chemical lipid extraction, and metabolic profiling provides the basis for using these techniques for the noninvasive presurgical metabolic assessment of $\mathrm{ccRCC}$ in patients and has the potential to overcome some of the limitations of percutaneous tumor biopsies in this heterogeneous disease. While intratumoral metabolic heterogeneity in ccRCC was confirmed and detected by Dixon-based MRI, our data demonstrates a higher degree of metabolic heterogeneity across different tumors.

\section{Methods}

Patient cohort and selection criteria. Patient selection for the study was performed according to the following criteria: known renal mass $\geq 2.5 \mathrm{~cm}$ scheduled for partial or radical nephrectomy, $>18$ years of age, and confirmed diagnosis of RCC at histopathology after surgical resection. Patients underwent MRI for evaluation of renal mass prior to surgery between August 2012 and March 2017. Among 83 patients, 62 patients had a ccRCC that was histologically confirmed after nephrectomy. Of these, 14 patients were excluded due to the lack of an FF map on MRI and 3 patients due to inability to do ROI analysis in predominantly cystic tumors. Exclusion criteria were non-ccRCC histology, patients not scanned with quantitative mDixon technique and/or FF reconstruction not available, and predominantly cystic tumors. Hence, 45 patients represent our study population (Supplemental Figure 2). The mean time between the MRI and the surgery was $5 \pm 4$ days (range 1-19 days).

Histopathology. Tumor specimens were oriented spatially using fiducial markers placed during surgery. The tumor specimen was then bivalved through the center of the mass to match the imaging plane of the MRI examination as previously described (53). The adequacy of colocalization of MRI images and specimen was assessed visually based on shape of the tumor and internal features (e.g., cystic degeneration, scar, necrosis) when possible. Tissue blocks were fixed in $10 \%$ buffered formaldehyde solution, sliced at 3-mm intervals, and embedded in paraffin. Tissue sections were sliced in $4 \mu \mathrm{m}$-thick sections and stained using H\&E; 4- $\mu \mathrm{m}$ sections were used for IHC. Histopathologic diagnosis was obtained by means of partial $(n=28)$, radical $(n=16)$, or simple $(n=1)$ nephrectomy (Supplemental Table 1$)$. All tumors were graded based on the ISUP guidelines (grades 1-4). Histopathologic analysis confirmed the diagnosis of ccRCC in these 45 patients; of those, 19 tumors were low grade (ISUP grade 1-2) ccRCC and 26 high grade (ISUP grade 3-4) ccRCC. Tumor samples for different imaging-tissue-based correlation analysis were obtained based on availability. A total of 27 fresh tumor samples matching the location of the tROI within FF map were obtained from 22 patients ( 2 samples from 3 patients, 3 samples from 1 patient) and stained with ORO. The percentage of cells that were stained with ORO was estimated visually to the nearest 5 th percentage (e.g. $5 \%, 10 \%, 15 \%$, etc.) by a urological pathologist who was blinded to the FF results. Histological slides of ORO were digitized with the optical image acquisition system Aperio Spectrum plus and Scanscope (Leica Biosystems). Lipid in renal tumor was clearly identified as punctate red droplets within the ORO-stained histology slides (Figure 2A). FASN monoclonal antibody (clone C20G5, Cell Signaling Technology) immunostaining was done on FFPE tumor sections using automated immunostainer (Dako North America Inc.) in the Kidney Cancer Core histology laboratory (UT Southwestern Medical Center, Dallas, Texas, USA) according to the manufacturer-recommended protocol, and the staining was analyzed using automated macros on Aperio Imagescope Software (Leica Biosystems). A total of 23 URP samples and 57 additional tumor samples (23 patients) were obtained. 
Based on tissue sample size, we used those samples for either metabolomic analysis alone (i.e., small samples) or for both metabolomic and lipidomic analysis (i.e., larger samples). Overall, 14/23 URP samples and 33/57 tumor samples were cut in half with one half used for lipidomic profile analysis and the other half for metabolomic analysis (see below). The rest of the URP (9/23) and tumor (24/57) samples were used for metabolomic analysis only. All tissue-based analyses were interpreted without previous knowledge of clinical data or MRI results.

MRI protocol. All patients were imaged in the supine position with a commercial 3T dual-transmit MR scanner with a 16-channel SENSE-XL-Torso coil (Achieva and Ingenia, Philips Healthcare). Axial and coronal T2-weighted half-Fourier single-shot turbo spin-echo (SShTSE) images were acquired for anatomic reference with the following imaging parameters: repetition time/echo time (TR/TE), 1,115/80 ms; flip angle (FA), 90\%; number of signal averages (NSA), 1; slice thickness, $5 \mathrm{~mm}$; field of view (FOV), $402 \times 340 \mathrm{~mm}^{2}$; matrix, $284 \times 268$; bandwidth, $467 \mathrm{~Hz} /$ pixel. Axial 3D T1W fast field-echo (FFE) mDixon quant (Philips Healthcare, Best, The Netherlands) acquisitions were then obtained to cover the entire renal mass with the following parameters: TR/TE, 6.7-8/1.09-1.24 ms; $\Delta \mathrm{TE}, 0.9-1.1 \mathrm{~ms}$; number of echoes, 6; FA, $2^{\circ}-3^{\circ}$; NSA, 1; slice thickness, $3 \mathrm{~mm}$; in-plane resolution, $1.5 \times 2 \mathrm{~mm}^{2}$; acquisition FOV, $402 \times 240 \times 96 \mathrm{~mm}^{3}$; acquisition matrix, $268 \times 120 \times 32$, bandwidth, $1,413 \mathrm{~Hz} /$ pixel. To minimize respiratory motion during Dixon MRI, patients held their breath for 15-19 seconds, dependent on the number of slices acquired. Patient respiratory motion was monitored with respiratory bellows, and good breath-holds were confirmed in all patients. These acquisitions where obtained as part of a more comprehensive multiparametric MRI examination including arterial spin labeling (ASL), diffusion weighted imaging (DWI), and dynamic contrast enhanced (DCE) MRI (53). For the purpose of this study, ASL, DWI, and DCE MRI data were not analyzed.

Image analysis. The FF map was reconstructed based on a multipeak model, with the frequency offsets $3.7,3.33,3.01,2.57,2.35,1.83$, and $-0.71 \mathrm{ppm}$ in the spectral model of the fat signal, which is relative to water frequency (54). For our analysis, we used the well-established multipeak fat spectrum model that has been validated in the liver (45) and extended to other sites including breast (54) and bone marrow (55). All images were analyzed on an iMac system (OS X; Apple Computer) equipped with an open-source digital imaging and communications in medicine (DICOM) viewer (Osirix X, version 5.6, 64 bit). The maximum size of the renal mass was measured on the axial or coronal T2-weighted images demonstrating the largest tumor dimension. Total tumor fat content was measured by a radiologist, who was blinded to all other results of the study, by placing an ROI including the entire tumor on each axial slice demonstrating the tumor on the $3 \mathrm{D} F F$ reconstruction. A 3D ROI of the whole-tumor FF was then created based on these slices, and the mean FF and SD within this $3 \mathrm{D}$ FF volume was recorded for each tumor. In order to assess the degree of intra- and intertumoral heterogeneity of fat in ccRCCs, 8 ROIs (approximately $0.5 \mathrm{~cm}^{2}$ in size) were placed systematically (sROIs) in each tumor, with 4 located in the upper half of the tumor and 4 in the lower half in the anteromedial, anterolateral, posteromedial, and posterolateral locations (for smaller tumors, only 2 ROIs in the upper half and 2 in the lower half were used). Additionally, a tROI was placed on representative locations on the FF map correlating to tissue samples obtained for ORO staining. Although magnetic susceptibility from hemorrhage is common in ccRCC, these Dixon methods are accurate for determination of FF even in the presence of iron overload (56). Visceral and subcutaneous fat with $>$ $90 \% \mathrm{FF}$ and the native kidneys with negligible $\mathrm{FF}(<3 \%)$ served as the internal reference for adequate fitting. No additional precautions were taken to correct the pixels with bad R2* fitting.

Mass spectrometry (lipidomic profile analysis). Lipids were measured by directly infusing a Blight-Dyer extracted sample into a SCIEX 5600+ TripleTOF MS (SCIEX) using MS/MS ${ }^{\text {ALL }}$ (commonly known as "shotgun lipidomics"). Cholesteryl esters were identified based on their product-ion mass of $369 \mathrm{Da}$. Free cholesterol was identified by the 369.3/148.2 Da mass combinations and confirmed by the 369.3/121.1 Da combinations. TG were identified based on their fatty acid neutral loss in positive ion mode using the LIPID MAPS database (http://www.lipidmaps.org/). The intensity of each peak was normalized to the total lipid signal and summed to give the intensity of each class, which was reported as the percentage of all identified lipids.

Metabolomics methods and analysis. Immediately after surgical resection, tumor specimens were placed in ice in the operating room and transferred to the Pathology Department, where each tumor was anatomically oriented using fiducial markers placed during surgery (Figure 1) and bivalved to match the imaging plane of the MRI examination (53). Targeted tissue fragments from tumor and URP were then obtained and were kept on ice and then transferred to $-80^{\circ} \mathrm{C}$ for storage. Each tissue 
fragment was homogenized with $80 \%$ methanol in water followed by three freeze-thaw cycles in liquid nitrogen. The insoluble material was pelleted in a cooled centrifuge $\left(4^{\circ} \mathrm{C}\right)$, and the supernatant was transferred to a new tube and evaporated to dryness using a SpeedVac concentrator (Thermo Savant). Metabolites were reconstituted in $100 \mathrm{ml}$ of $0.03 \%$ formic acid in analytical-grade water, vortex-mixed, and centrifuged to remove debris. Samples were then injected in randomized order onto an AB SCIEX QTRAP 5500 liquid chromatograph/triple quadrupole mass spectrometer.

Separation was achieved on a Phenomenex Synergi Polar-RP HPLC column $(150 \times 2 \mathrm{~mm}, 4 \mu \mathrm{m}$, $80 \AA$ A) using a Nexera Ultra High Performance Liquid Chromatograph (UHPLC) system (Shimadzu Corporation). The mobile phases employed were $0.03 \%$ formic acid in water (A) and $0.03 \%$ formic acid in acetonitrile (B). The gradient program was as follows: $0-3 \mathrm{~min}, 0 \% \mathrm{~B} ; 3-15 \mathrm{~min}, 0 \%-100 \% \mathrm{~B}$; 15-17 $\mathrm{min}, 100 \% \mathrm{~B} ; 17-17.1 \mathrm{~min}, 100 \%-0 \% \mathrm{~B} ; 17.1-20 \mathrm{~min}, 0 \% \mathrm{~B}$. The column was maintained at $35^{\circ} \mathrm{C}$ and the samples kept in the autosampler at $4^{\circ} \mathrm{C}$. The flow rate was $0.5 \mathrm{ml} / \mathrm{min}$, and the injection volume was $20 \mu 1$. The mass spectrometer was an AB QTRAP 5500 (Applied Biosystems, SCIEX) with electrospray ionization (ESI) source in multiple reaction monitoring (MRM) mode. Sample analysis was performed in positive/negative switching mode. Declustering potential (DP), collision energy, and collision cell exit potential (CXP) were optimized for each metabolite by direct infusion of reference standards using a syringe pump prior to sample analysis. The MRM tandem mass spectrometry detector conditions were set as follows: curtain gas $30 \mathrm{psi}$; ion spray voltages 1,200 V (positive) and -1,500 $\mathrm{V}$ (negative); temperature $650^{\circ} \mathrm{C}$; ion source gas 1 at 50 psi and ion source gas 2 at 50 psi (arbitrary pressure units on 0-90 scale); interface heater on; entrance potential $10 \mathrm{~V}$. Dwell time for each transition was set at $3 \mathrm{~ms}$. Samples were analyzed in a randomized order, and MRM data was acquired using Analyst 1.6.1 software (Applied Biosystems SCIEX, Foster City, CA).

Chromatogram review and peak area integration were performed using MultiQuant software version 2.1 (Applied Biosystems, SCIEX). Although the numbers of cells were similar and each sample was processed identically and randomly, the peak area for each detected metabolite was normalized against the protein content of that sample to correct any variations introduced from sample handling through instrument analysis. The normalized area values were used as variables for the multivariate and univariate statistical data analysis. The chromatographically coeluted metabolites with shared MRM transitions were shown in a grouped format (i.e., leucine/isoleucine). All multivariate analyses and modeling on the normalized data were carried out using SIMCA-P (version 13.0.1, Umetrics). The preprocessed datasets were mean-centered and unit-variance scaled and were then evaluated by PCA to visualize the clustering trend, as well as to detect and exclude outlier datasets. Univariate statistical differences of the metabolites between two groups were analyzed using two-tailed Student's $t$ test adjusted by Benjamini-Hochberg procedures. Adjusted $P$ values less than 0.05 were considered as statistically significant.

Statistics. Mean FF and SD for 3D ROIs were correlated to tumor size and ISUP grade. The Spearman rank-order correlation was used to assess the correlation between FF from Dixon MRI and ORO percentage, and between FF from Dixon MRI and lipidomic profile. Spearman correlation coefficient and the 95\% bootstrap CI were also calculated after adjusting for repeated measurements in the same tumor (i.e., more than one tissue sample per tumor). A linear regression model was also performed to establish the relation between FF and ORO percentage. The ICC of the sROIs was calculated to determine the effect of intra- and intertumor heterogeneity in FF. To assess the effect of tumor size in intratumor heterogeneity, the SD of whole-tumor FF and maximum tumor dimension and volume were correlated with Spearman rank-order correlation. Two one-sided Wilcoxon rank sum tests were used to test the alternative hypothesis that grade- 4 tumors have lower median FF than grade-2 and -3 tumors. All statistical analyses were performed with Prism software package (Version 6.05, GraphPad Software Inc.) with $P<0.05$ considered statistically significant.

Study Approval. The UT Southwestern Medical Center IRB approved this Health Insurance Portability and Accountability Act-compliant (HIPAA-compliant) prospective study. A written informed consent was obtained from all patients before imaging.

\section{Author contributions}

Study design contributed by IP; imaging study and analysis contributed by YZ, IP, QY, IED, TY, ADDL, DU, DKD, AJM, REL, MF; metabolic analysis contributed by ZH, EYK, LC, RJD; lipid mass spectometry studies contributed by JY, MAM, HK, JGM; histopathology contributed by PK, APJ, JAC, VM, JB, DU, YZ, IP; statistics contributed by YZ, LC, YX; and all author assisted in manuscript draft. 


\section{Acknowledgments}

The authors would like to thank Trevor Wigal, Shiju Varughese, and Christina Carrigan for patient recruitment and MRI scans. We thank Farrah Homayoun for her technical assistance with the histology analysis. Funding was received from NIH R01CA154475 (YZ, MF, IP), NIH P50CA196516 (IP, JB, RD, JAC, PK), Welch Foundation I-1832 (JY), and NIH P01HL020948 (JGM).

Address correspondence to: Ivan Pedrosa, 5323 Harry Hines Boulevard, Dallas, Texas 75390-9085, USA. Phone: 214.645.7386, Email: Ivan.Pedrosa@UTSouthwestern.edu.

JGM's present address is: Center for Human Nutrition, University of Texas Southwestern Medical Center, Dallas, Texas, USA.

1. Motzer RJ, Bukowski RM. Targeted therapy for metastatic renal cell carcinoma. J Clin Oncol. 2006;24(35):5601-5608.

2. Cheville JC, Lohse CM, Zincke H, Weaver AL, Blute ML. Comparisons of outcome and prognostic features among histologic subtypes of renal cell carcinoma. Am J Surg Pathol. 2003;27(5):612-624.

3. Teloken PE, et al. Prognostic impact of histological subtype on surgically treated localized renal cell carcinoma. $J$ Urol. 2009;182(5):2132-2136.

4. Jonasch E, et al. State of the science: an update on renal cell carcinoma. Mol Cancer Res. 2012;10(7):859-880.

5. Kaelin WG. Treatment of kidney cancer: insights provided by the VHL tumor-suppressor protein. Cancer. 2009;115(10 Suppl):2262-2272.

6. Kim W, Kaelin WG. The von Hippel-Lindau tumor suppressor protein: new insights into oxygen sensing and cancer. Curr Opin Genet Dev. 2003;13(1):55-60.

7. Cancer Genome Atlas Research Network. Comprehensive molecular characterization of clear cell renal cell carcinoma. Nature. 2013;499(7456):43-49.

8. Krishnan B, Truong LD. Renal epithelial neoplasms: the diagnostic implications of electron microscopic study in 55 cases. Hum Pathol. 2002;33(1):68-79.

9. Horiguchi A, Asano T, Asano T, Ito K, Sumitomo M, Hayakawa M. Fatty acid synthase over expression is an indicator of tumor aggressiveness and poor prognosis in renal cell carcinoma. J Urol. 2008;180(3):1137-1140.

10. von Roemeling CA, et al. Stearoyl-CoA desaturase 1 is a novel molecular therapeutic target for clear cell renal cell carcinoma. Clin Cancer Res. 2013;19(9):2368-2380.

11. Gebhard RL, et al. Abnormal cholesterol metabolism in renal clear cell carcinoma. J Lipid Res. 1987;28(10):1177-1184.

12. Tugnoli V, et al. 1H-NMR and 13C-NMR lipid profiles of human renal tissues. Biopolymers. 2003;72(2):86-95.

13. Tomaszewski JJ, Uzzo RG, Smaldone MC. Heterogeneity and renal mass biopsy: a review of its role and reliability. Cancer Biol Med. 2014;11(3):162-172.

14. Gerlinger $\mathrm{M}$, et al. Intratumor heterogeneity and branched evolution revealed by multiregion sequencing. $N$ Engl $J$ Med. 2012;366(10):883-892.

15. Gerlinger M, et al. Genomic architecture and evolution of clear cell renal cell carcinomas defined by multiregion sequencing. Nat Genet. 2014;46(3):225-233.

16. Eggers H, Brendel B, Duijndam A, Herigault G. Dual-echo Dixon imaging with flexible choice of echo times. Magn Reson Med. 2011;65(1):96-107.

17. Bannas P, et al. Quantitative magnetic resonance imaging of hepatic steatosis: Validation in ex vivo human livers. Hepatology. 2015;62(5):1444-1455.

18. Tang A, et al. Accuracy of MR imaging-estimated proton density fat fraction for classification of dichotomized histologic steatosis grades in nonalcoholic fatty liver disease. Radiology. 2015;274(2):416-425.

19. Idilman IS, et al. A comparison of liver fat content as determined by magnetic resonance imaging-proton density fat fraction and MRS versus liver histology in non-alcoholic fatty liver disease. Acta Radiol. 2016;57(3):271-278.

20. Delahunt B, et al. The International Society of Urological Pathology (ISUP) grading system for renal cell carcinoma and other prognostic parameters. Am J Surg Pathol. 2013;37(10):1490-1504.

21. Notarnicola M, Tutino V, Caruso MG. Tumor-induced alterations in lipid metabolism. Curr Med Chem. 2014;21(24):2729-2733

22. Drabkin HA, Gemmill RM. Cholesterol and the development of clear-cell renal carcinoma. Curr Opin Pharmacol. 2012;12(6):742-750.

23. Zhang F, Du G. Dysregulated lipid metabolism in cancer. World J Biol Chem. 2012;3(8):167-174.

24. Reeder SB, et al. Multicoil Dixon chemical species separation with an iterative least-squares estimation method. Magn Reson Med. 2004;51(1):35-45.

25. Kruth HS. Localization of unesterified cholesterol in human atherosclerotic lesions. Demonstration of filipin-positive, oil-redO-negative particles. Am J Pathol. 1984;114(2):201-208.

26. Börnig H, Geyer G. Staining of cholesterol with the fluorescent antibiotic "filipin". Acta Histochem. 1974;50(1):110-115.

27. Ramírez-Zacarías JL, Castro-Muñozledo F, Kuri-Harcuch W. Quantitation of adipose conversion and triglycerides by staining intracytoplasmic lipids with Oil red O. Histochemistry. 1992;97(6):493-497.

28. Wettersten HI, et al. Grade-Dependent Metabolic Reprogramming in Kidney Cancer Revealed by Combined Proteomics and Metabolomics Analysis. Cancer Res. 2015;75(12):2541-2552.

29. Saito K, et al. Lipidomic Signatures and Associated Transcriptomic Profiles of Clear Cell Renal Cell Carcinoma. Sci Rep. 2016;6:28932.

30. Yoshimura K, et al. Analysis of renal cell carcinoma as a first step for developing mass spectrometry-based diagnostics. $J$ Am Soc 
Mass Spectrom. 2012;23(10):1741-1749.

31. Hoffmann K, Blaudszun J, Brunken C, Höpker WW, Tauber R, Steinhart H. Lipid class distribution of fatty acids including conjugated linoleic acids in healthy and cancerous parts of human kidneys. Lipids. 2005;40(10):1057-1062

32. Beloribi-Djefaflia S, Vasseur S, Guillaumond F. Lipid metabolic reprogramming in cancer cells. Oncogenesis. 2016;5:e189.

33. Menendez JA, Lupu R. Fatty acid synthase and the lipogenic phenotype in cancer pathogenesis. Nat Rev Cancer. 2007;7(10):763-777

34. Kuhajda FP. Fatty acid synthase and cancer: new application of an old pathway. Cancer Res. 2006;66(12):5977-5980.

35. Li B, et al. Fructose-1,6-bisphosphatase opposes renal carcinoma progression. Nature. 2014;513(7517):251-255.

36. Hakimi AA, et al. An Integrated Metabolic Atlas of Clear Cell Renal Cell Carcinoma. Cancer Cell. 2016;29(1):104-116.

37. Lucarelli G, et al. Metabolomic profile of glycolysis and the pentose phosphate pathway identifies the central role of glucose-6-phosphate dehydrogenase in clear cell-renal cell carcinoma. Oncotarget. 2015;6(15):13371-13386.

38. Hensley CT, et al. Metabolic Heterogeneity in Human Lung Tumors. Cell. 2016;164(4):681-694.

39. National Comprehensive Cancer Network (NCCN) Clinical Practice Guideines in Oncology. Kidney Cancer http://www.nccn. org/professionals/physician_gls/pdf/kidney.pdf Version 2.2017.Updated October 31, 2016. Accessed July $28,2017$.

40. Campbell N, Rosenkrantz AB, Pedrosa I. MRI phenotype in renal cancer: is it clinically relevant? Top Magn Reson Imaging. 2014;23(2):95-115.

41. Pedrosa I, Alsop DC, Rofsky NM. Magnetic resonance imaging as a biomarker in renal cell carcinoma. Cancer. 2009;115(10 Suppl):2334-2345

42. Walther TC, Farese RV. Lipid droplets and cellular lipid metabolism. Annu Rev Biochem. 2012;81:687-714.

43. Pedrosa I, et al. MR classification of renal masses with pathologic correlation. Eur Radiol. 2008;18(2):365-375.

44. Yoshimitsu K, et al. MR detection of cytoplasmic fat in clear cell renal cell carcinoma utilizing chemical shift gradient-echo imaging. J Magn Reson Imaging. 1999;9(4):579-585.

45. Meisamy S, et al. Quantification of hepatic steatosis with T1-independent, T2-corrected MR imaging with spectral modeling of fat: blinded comparison with MR spectroscopy. Radiology. 2011;258(3):767-775.

46. Yokoo T, et al. Quantification of renal steatosis in type II diabetes mellitus using dixon-based MRI. J Magn Reson Imaging. 2016;44(5):1312-1319.

47. Dennis EA, et al. A mouse macrophage lipidome. J Biol Chem. 2010;285(51):39976-39985.

48. Leonardi R, Frank MW, Jackson PD, Rock CO, Jackowski S. Elimination of the CDP-ethanolamine pathway disrupts hepatic lipid homeostasis. J Biol Chem. 2009;284(40):27077-27089.

49. Pal SK, Haas NB. Adjuvant therapy for renal cell carcinoma: past, present, and future. Oncologist. 2014;19(8):851-859.

50. Mehrazin R, et al. Growth kinetics and short-term outcomes of cT1b and cT2 renal masses under active surveillance. $J$ Urol. 2014;192(3):659-664

51. Horng DE, Hernando D, Hines CD, Reeder SB. Comparison of R2* correction methods for accurate fat quantification in fatty liver. J Magn Reson Imaging. 2013;37(2):414-422.

52. Trivedi $\mathrm{H}$, et al. Use of patient-specific MRI-based prostate mold for validation of multiparametric MRI in localization of prostate cancer. Urology. 2012;79(1):233-239.

53. Zhang Y, et al. Tumor Vascularity in Renal Masses: Correlation of Arterial Spin-Labeled and Dynamic Contrast-Enhanced Magnetic Resonance Imaging Assessments. Clin Genitourin Cancer. 2016;14(1):e25-e36.

54. Dimitrov IE, et al. In vivo determination of human breast fat composition by ${ }^{1} \mathrm{H}$ magnetic resonance spectroscopy at $7 \mathrm{~T}$. Magn Reson Med. 2012;67(1):20-26.

55. Karampinos DC, et al. Modeling of T2* decay in vertebral bone marrow fat quantification. NMR Biomed. 2015;28(11):1535-1542.

56. Horng DE, Hernando D, Reeder SB. Quantification of liver fat in the presence of iron overload. J Magn Reson Imaging. 2017;45(2):428-439. 